\title{
Attentes et promesses du risque de psychose
}

\author{
Nicolas Henckes ${ }^{a}$ \\ a. Chargé de Recherche CNRS \\ CERMES3 - Centre de Recherche Médecine, Sciences, Santé, Santé mentale et Société - \\ CNRS UMR8211 - INSERM U988 - EHESS - UPD \\ 7 rue Guy Môquet \\ 94801 Villejuif Cedex \\ nicolas.henckes@cnrs.fr
}

Version pré-publication de : Henckes, Nicolas. "Attentes et promesses du risque de psychose." L'Evolution Psychiatrique 81, no. 1 (2016): 43-52.

\section{Résumé}

Objectif. La création à la fin des années 1990 de la catégorie d'état mental à risque pour caractériser des présentations cliniques susceptibles d'évoluer à échéance de quelques mois vers la psychose est à l'origine d'une nouvelle psychiatrie du jeune adulte, organisée notamment autour du dépistage et de la prise en charge de ces états. Cet article analyse les attentes et les promesses de cette nouvelle psychiatrie.

Méthode. L'article mobilise une approche de sociologie de la connaissance mettant en évidence les intérêts, les contextes institutionnels et les pratiques qui contribuent à façonner les savoirs et catégories psychiatriques ainsi que les débats que ces savoirs et catégories font naître.

Résultats. Les débats et recherches sur les états mentaux à risque au cours des vingt dernières années ont considérablement fait évoluer la vision des cliniciens sur cette catégorie. Les difficultés à stabiliser le risque de transition psychotique chez les patients concernés ont amené les promoteurs de cette catégorie à en proposer une interprétation nouvelle : sans être le signe nécessairement d'une évolution vers la psychose, les états mentaux à risque seraient indicatifs d'un devenir pathologique à la fois ouvert dans ses développements et certain dans son accomplissement. La communauté psychiatrique est divisée entre deux façons d'aborder ces résultats : affiner la catégorie pour la rendre réellement prédictive ou en faire le point de départ d'une nouvelle stratégie de santé publique orientée vers la santé mentale du jeune adulte.

Discussion. Les états mentaux à risque sont à l'origine d'une clinique de l'attente paradoxale caractérisée par l'indétermination des perspectives et des promesses qui y sont attachées. C'est cette indétermination qui pourrait expliquer son succès auprès d'un large ensemble d'acteurs issus de la clinique, de la recherche et de la politique aux intérêts divers.

Conclusion. Une meilleure compréhension des dynamiques d'innovation et de réforme en psychiatrie devrait prêter attention aux conditions sociales des promesses psychiatriques.

Mots-clefs : Psychose; Nosographie; Sociologie; Vulnérabilité 


\section{Attentes et promesses du risque de psychose}

Nicolas Henckes

Depuis une vingtaine d'années une nouvelle psychiatrie du jeune adulte a émergé autour de la promesse d'une prévention des maladies mentales et de la perspective d'une réforme globale de la psychiatrie. Si elle prétend aujourd'hui apporter une réponse aux problèmes que soulève la santé mentale des jeunes adultes dans l'ensemble de ses dimensions, cette psychiatrie reste structurée autour d'un ensemble de pratiques cliniques et de travaux de recherche visant une catégorie spécifique : les " états mentaux à risque ». Créée par des psychiatres australiens à la fin des années 1990 cette catégorie visait à caractériser des présentations cliniques susceptibles d'évoluer à échéance de quelques mois vers la schizophrénie. (Yung et al., 1996) D’abord constituée en objet de recherche clinique, elle figure aujourd'hui au centre d'un nombre croissant d'initiatives issues de la clinique, de la recherche fondamentale et des politiques de santé mentale qui en font l'un des concepts les plus discutés de la psychiatrie mondiale aujourd'hui. Si les méthodes, techniques et approches des neurosciences sont un élément important de la science des états mentaux à risque, celle-ci mobilise également des savoirs issus de la génétique et de l'épidémiologie en passant par la phénoménologie, tandis que, sur le plan clinique, les acteurs s'appuient sur des approches thérapeutiques allant de la psychopharmacologie aux thérapies cognitivo-comportementale en passant par le conseil et le travail social. Malgré quelques initiatives sur le terrain et un début d'intérêt dans la littérature, cette psychiatrie a encore peu de retentissement en France pour des raisons qui tiennent vraisemblablement à la fois à des traditions cliniques, à un héritage politique et professionnel, autant qu'aux orientations et à l'organisation de la recherche notamment clinique (Krebs et Canceil, 2004; Petitjean et Marie-Cardine, 2003). Elle s'est cependant imposée dans un grand nombre de pays développés comme l'une des perspectives les plus fécondes de transformation de la discipline.

Cet article explore l'assemblage de savoirs, institutions et politiques au cœur de cette nouvelle psychiatrie. Les deux concepts centraux de l'analyse sont ceux d'attentes et de promesses : attentes intrinsèques aux états mentaux à risque et promesses dont ceux-ci sont porteurs pour la psychiatrie. Ces dernières années une riche sociologie des anticipations s'est constituée, notamment autour de la notion de risque, pour interroger les façons dont certains futurs en viennent à être inscrits dans nos présents. Cette sociologie a notamment mis en évidence les régimes d'action spécifiques qui s'organisent autour de ces anticipations. A cet égard la psychiatrie du jeune adulte constituée autour des états mentaux à risque se caractérise par la construction de projections qui rendent les futurs à la fois excessivement présents tout en les maintenant largement indéterminés. Il ne s'agit pas ici de proposer une critique de cette construction et de cette psychiatrie mais bien d'en donner quelques 
clefs d'analyse. De ce point de vue les tensions générées par cette présence et cette indétermination sont l'une des clefs de compréhension des transformations de la clinique des psychoses aujourd'hui. ${ }^{1}$

\section{Une clinique de l'attente}

Depuis les premières définitions à la fin des années 1990, plusieurs concepts et ensembles de critères ont été développés pour caractériser les états mentaux à risque et il n'y actuellement pas même consensus sur leur dénomination, au point que certains observateurs en sont venus à déplorer la confusion babélienne qui règne sur ces questions. Ces critères se rejoignent cependant pour la plupart en ce qu'ils mettent au centre de leur caractérisation des symptômes psychotiques positifs atténués ainsi qu'un critère d'âge, le plus souvent de 16 à 30 ans. Les états mentaux à risque correspondraient ainsi, d'un point de vue phénoménal, à des psychoses en gestation chez des jeunes adultes. La représentation la plus courante dans la littérature est celle d'un processus parcourant une trajectoire continue l'amenant à passer ou non certains seuils de significativité clinique jusqu'à la maladie déclarée, l'ensemble étant rendu par une courbe dans un espace à deux dimensions. (cf. fig. 1)

\section{Insérer la Fig. 1 ici}

Il y aurait beaucoup à dire sur ces courbes, ce qu'elles supposent et ce qu'elles impliquent, leurs usages et leur efficacité performative. $S^{\prime}$ il ne fait aucun doute qu'elles réduisent considérablement la complexité d'un phénomène dont la nature est un mystère et la description même reste problématique, on peut penser que c'est précisément ce réductionnisme qui a été une des clefs du succès des états mentaux à risque. Non seulement ces courbes donnent à voir un processus sur lequel il est dès lors envisageable d'agir, mais elles organisent aussi des façons de penser à son sujet. Loin d'être seulement des illustrations à vocation pédagogique, ces courbes sont en effet des modèles qui ont joué un rôle central dans le développement des états mentaux à risque et qui continuent de structurer la réflexion à leur sujet. Au cours des dernières années c'est ainsi sur un point précis de ces courbes que se sont concentrées les discussions : la transition psychotique, soit le risque pour les patients répondant aux critères de la catégorie de développer une psychose. Autrement dit, sur les attentes attachées à la catégorie.

\section{L’objectivité du risque}

Dans un premier temps c'est la confiance qu'il fallait mettre dans ces attentes qui a été mise en question. Le problème est venu des difficultés à stabiliser un taux de transition psychotique unique dans les différentes études menées sur des échantillons de patients à risque. Alors que les premières d'entre elles suggéraient un taux de transition de $40 \%$ à 12 mois (Yung et al., 2003), leur réplication au milieu des années 2000 dans les centres mêmes où elles avaient été menées aboutissaient à des taux inférieurs (Yung et al., 2007). La méta-analyse publiée en 2012 par Fusar-Poli et collègues à partir de 27 études, actuellement considérée comme l'état de l'art sur la question, montrait que le déclin du taux de transition au cours du temps était une tendance statistiquement significative.

(Fusar-Poli et al., 2012)

\footnotetext{
${ }^{1}$ Cet article s'appuie sur une série de recherches menées dans le cadre du programme « Psychiatric Fringes. » en collaboration avec l'équipe de l'institut d'histoire de la médecine de la Charité, Berlin, et avec des financements de l'ANR, la DFG, le CNRS et la Ville de Paris.
} 
De nombreuses explications ont été avancées pour expliquer ce phénomène sans qu'aucune s'impose réellement (Yung et al., 2007) : des changements dans les critères utilisés, une amélioration des prises en charge, ou encore une évolution du recrutement des centres à mesure qu'ils devenaient mieux connus. Quelles que soient leur limites, ces explications pointent néanmoins vers une hypothèse : le caractère prédictif de la catégorie tient peut-être moins aux échelles elles-mêmes qu'au dispositif encadrant le recrutement des patients. (Van Os et Delespaul, 2005) C'est parce que les patients sont passés par le filtre d'un réseau d'adressage qu'il est possible, en fin de parcours, de constituer des échantillons présentant un risque très élevé de psychose. D’où les difficultés à utiliser les échelles en population générale : sans ce filtre, la prédiction est impossible. Les patients à risque seraient dans cette perspective non seulement des patients répondant aux critères de risque mais aussi des patients vus dans des services de détection des états à risque. S'il est impossible d'imaginer une sémiologie sans le support institutionnel qui la fait émerger (Lantéri-Laura, 1993), les états mentaux à risque seraient ainsi un cas limite où l'institution constitue l'objectivité de la catégorie. Cela ne signifie pas que celle-ci perde tout intérêt, mais cela rend le problème de la maîtrise des taux de transition plus complexe.

\section{Vers une sémiologie distale}

L'absence de solution pratique à ce problème a ainsi conduit ces dernières années la discussion à se déplacer à un autre niveau : celui du contenu des attentes attachées à la catégorie elles-mêmes. Un élément contextuel a également contribué à cette évolution : le débat suscité de 2010 à 2013 par l'inclusion éventuelle dans le DSM5 d'un « syndrome de risque de psychose " modelé d'après les états mentaux à risque. Se voyant opposer la faible prédictibilité de la catégorie, les tenants de l'inclusion en vinrent à avancer plusieurs arguments pour repenser son utilité clinique. Le premier est la variabilité des présentations cliniques pouvant satisfaire aux critères d'état mental à risque, conduisant les uns à parler de comorbidités, les autres à suggérer que les symptômes psychotiques seraient un signe susceptible de se surajouter à une diversité d'états pathologiques. Quoi qu'il en soit de ce dernier point, les états mentaux à risque apparaitraient ainsi comme une porte ouverte sur une psychopathologie spécifique du jeune adulte justifiant qu'on la prenne mieux en compte. En second lieu, certains cliniciens se mirent à suggérer que la transition psychotique n'était pas la seule raison de prendre en charge les patients à risque : ces derniers sont symptomatiques, ont une demande et, le plus souvent, "fonctionnent mal ", de sorte que même sans répondre aux critères d'une entité clinique caractérisée, ils pourraient bénéficier d'une prise en charge. (Ruhrmann et al., 2010) Au final c'est comme on sait cet argument qui emporta l'adhésion, conduisant les rédacteurs du DSM5 à inclure dans une annexe la catégorie de syndrome psychotique atténué. Ce développement appelle deux commentaires.

Le premier est que les états mentaux à risque paraissent ainsi pouvoir être rapprochés d'autres états aux limites de la psychopathologie qui se caractérisent par des présentations cliniques ambiguës et dont la clinique n'explique pas seule la significativité. On pense par exemple aux troubles dépressifs anxieux mixtes de la CIM10, dont des études épidémiologiques menées en Grande Bretagne ont montré qu'ils constituent l'un des premiers motifs d'arrêt de travail pour raison de santé mentale dans ce pays. (Das-Munshi et al., 2008) S'ils ne correspondent pas à des états cliniques constitués, les états mentaux à risque, comme les troubles dépressifs anxieux mixtes, caractériseraient ainsi des présentations au retentissement fonctionnel suffisamment important pour qu'on ait à s'en préoccuper. On verrait ainsi se constituer une clinique du fonctionnement, qui ressortirait à une 
clinique déjà riche de l'infra pathologique autour notamment de la souffrance psychique ou du traumatisme. Si l'hypothèse est séduisante il faut cependant peut-être se garder de la prendre au pied de la lettre. La distinction entre fonctionnement et symptôme mériterait une discussion approfondie mais il est clair qu'elle est moins solide d'un point de vue théorique qu'il pourrait y paraître au premier abord et qu'il est difficile de penser une bonne part de la sémiologie sans le recours à des concepts de fonctionnement ou de handicap. (Wakefield, 2009) Dans ces conditions c'est plutôt une nouvelle sémiologie qui serait en jeu : fondée non pas sur des signes exprimant directement un processus pathologique mais plutôt sur des « symptômes distaux » reflet d'un trouble que la clinique ne sait ni observer directement, ni caractériser précisément mais qui n'en est pas moins là ${ }^{2}$.

\section{Des attentes sans objet}

A cette discussion, les états mentaux à risque ajoutent un élément de complexité supplémentaire. Car l'argument selon lequel ils correspondraient à un état pathologique déjà là n'a pas conduit à remiser l'idée qu'ils seraient prédictifs d'autre chose. Pour ses promoteurs, l'intérêt des états mentaux à risque reste bien la prise qu'ils paraissent offrir sur une symptomatologie en développement. Pour autant, les incertitudes sur la transition psychotique les conduisent maintenant généralement à mettre l'accent l'indétermination de ces développements. Le même tableau clinique pourrait évoluer vers un trouble psychotique, un trouble de l'humeur ou un trouble anxieux. Dans ces conditions, l'enjeu pour les cliniciens est de proposer des prises en charge adaptées aux présentations actuelles en prêtant attention à leur évolutivité ${ }^{3}$. Dans un récent consensus, les principales figures du champ comparent ainsi les signes psychotiques atténués à une douleur de poitrine, susceptible d'évoluer vers un infarctus du myocarde comme d'être le symptôme d'une côte cassé. (Yung et al., 2012) Si l'image peut faire sourire par la vision quelque peu frustre qu'elle donne du diagnostic différentiel de la douleur de poitrine, elle reflète bien l'ampleur des incertitudes qui traverse aujourd'hui la détermination de la nature exacte des symptômes psychotiques atténués.

Si l'on prend au sérieux ces perspectives, les états mentaux à risque paraissent ainsi nourrir une clinique de l'attente paradoxale : tournée non pas vers la prédiction et l'anticipation de pathologies qui se déclareront probablement, mais bien plutôt vers la perspective d'un devenir à la fois ouvert dans ses développements et certain dans son accomplissement. A bien des égards ce sont des attentes sans objet que cette clinique paraît faire émerger.

\section{Les promesses des états mentaux à risque}

Comment comprendre les promesses dont cette clinique a paru être porteuse pour la psychiatrie ? Depuis la fin des années 1990 et les premières publications sur les états mentaux à risque, un riche milieu de chercheurs, cliniciens et activistes du monde entier s'est de fait progressivement constitué pour former aujourd'hui une communauté parmi les plus actives à l'échelle internationale dans le champ des psychoses. Les participants à cette communauté se sont lancés dans la création de services de prise en charge, le développement de projets de recherche clinique et fondamentale ou encore la promotion de politiques publiques. Pour organiser ce milieu, une association internationale

\footnotetext{
${ }^{2}$ L'expression de symptômes distaux est emprunté au travail de David Armstrong et collègues sur la notion de qualité de vie : (Armstrong et al., 2007)

${ }^{3}$ C'est la philosophie du modèle de « clinical staging » défendu par une partie de la communauté dans la suite de l'un de ses principaux leaders, Patrick McGorry (McGorry, 2007).
} 
a été créée dès 1998 pour et un journal en 2007. Localement, un certain nombre d'associations ou de réseaux se sont constituées à l'échelle nationale pour structurer les initiatives. En 2012, les principaux animateurs de cette communauté ont cherché à donner un nouvel essor au mouvement en lançant une seconde association, l'association internationale pour la santé mentale des jeunes, qui vise à créer un mouvement social autour des approches issues de leurs travaux et à en assurer la promotion auprès des décideurs.

Il est difficile de donner une idée quantitative de ces développements en dehors des indicateurs bibliométriques que l'on peut facilement constituer à partir des bases de données sur internet. S'il existe quelques enquêtes locales ou nationales elles donnent difficilement la mesure de l'influence actuelle du mouvement. Il paraît cependant certain qu'au-delà du noyau dur des pionniers et de leurs successeurs immédiats les états mentaux à risque suscitent un intérêt large dans la psychiatrie, que celui-ci soit critique ou au contraire d'adhésion. L'ampleur prise par le débat sur l'inclusion du syndrome de risque de psychose dans le DSM5 dans le monde entier montre combien celui-ci est devenu un enjeu central pour la discipline : à bien des égards, le risque de psychose est devenu un terrain privilégié pour repenser à la fois la clinique, la recherche et les politiques publiques en direction des psychoses. En dehors de la communauté psychiatrique, les promoteurs de la catégorie ont trouvé des relais au sein de certains think tanks mais aussi d'un mouvement social constitué notamment d'associations de jeunes issues des campus universitaires nord-américains. En quelques années c'est un agenda à la fois scientifique et politique qui s'est ainsi imposé.

\section{Deux approches des états mentaux à risque}

Si cette communauté est internationale, tant son organisation que ses perspectives diffèrent cependant selon les pays. On peut ainsi schématiquement distinguer deux approches différentes de la clinique, la recherche et la politique des états mentaux à risque qui permettent de classer les différentes communautés nationales. La première se structure autour de la poursuite du projet initial de prévention spécifique de la schizophrénie. Les tenants de cette approche se proposent d'affiner les critères pour constituer une catégorie réellement prédictive. L'un des enjeux, dans cette perspective, est de mettre en évidence des biomarqueurs à l'aide de la génétique ou de l'imagerie. Cette approche est dominante dans certains pays comme l'Allemagne, les Pays-Bas, la Suisse où dans une moindre mesure la France, où les états mentaux à risque sont restés à l'état de catégorie de recherche et ne sont pas sortis des cliniques universitaires, et elle est largement représentée aux USA ou en Grande Bretagne.

Dans ces deux pays et dans d'autres comme l'Australie, les états mentaux à risque sont par ailleurs également au cœur d'un mouvement social qui vise à faire avancer la catégorie sur l'agenda de la santé publique et plus largement à fonder des initiatives en faveur des jeunes adultes. En Australie les services mis en place par les promoteurs de la catégorie ont été promus tête de pont d'une politique de santé mentale largement renouvelée. En Grande Bretagne l'intervention précoce est devenu un axe prioritaire du National Health Service en matière de psychiatrie et ses promoteurs lancent maintenant des initiatives en direction des jeunes adultes en décrochage scolaire ou sans emploi.

\section{Des programmes très ouverts}


Loin de s'opposer, ces deux approches se renforcent en fait mutuellement au sein de la communauté internationale des états mentaux à risque, la perspective d'applications en santé publique constituant une justification implicite pour la recherche clinique et fondamentale. De façon marquante cette association nourrit chez les animateurs de la communauté un rapport spécifique à la preuve qui s'exprime dans les éditoriaux, opinions ou la littérature grise. Lorsqu'une revue de la littérature en vint à conclure que les interventions sur les états mentaux à risque ne reposaient pas sur des preuves d'un niveau suffisant (Marshall et Rathbone, 2011), c'est ainsi en termes de l'urgence d'agir alors que " l'on sait » que les services actuels sont caractérisés par un fonctionnement non optimal que le principal leader du mouvement a répondu à ses détracteurs. (McGorry, 2012) II peut être tentant d'interpréter cette réponse comme un argument stratégique dans une situation de blocage. Elle paraît plutôt correspondre à une façon spécifique de comprendre le rapport entre action politique et preuve scientifique : si les politiques de santé mentales doivent bien s'appuyer sur des preuves, l'absence de preuve ne doit pourtant pas être un obstacle à l'action mais bien une motivation pour la réforme, qui peut se nourrir d'autres impulsions et doit apporter ses propres preuves en se faisant. (McGorry, 2010)

Encore une fois il n'y a pas malice à relever le caractère quelque peu contourné de cet argument. Celui-ci paraît plutôt refléter à la fois les enjeux et la nature particulière des promesses dont les états mentaux à risque sont aujourd'hui porteurs. C'est en effet rien moins qu'une solution au problème de la psychose qui est en jeu. L'adhésion d'une partie significative du monde de la santé mentale à ces promesses participe ainsi clairement d'une forme de millénarisme tandis que l'on peut lire derrière ces dernières une forme de " pensée proleptique » selon l'expression de l'historien des neurosciences Cornelius Borck (Borck, 2009) : de même que les neurosciences n'ont eu de cesse de promettre un futur où la nature de l'esprit serait élucidée, pour être toujours détrompées sur les chemins par lequel ce futur serait atteint faute d'avoir pu sortir d'un cadre de pensée contingent de l'état de la recherche, la psychiatrie du jeune adulte décrit l'avènement prochain d'un futur où les mystères de la psychose seront enfin percés. La comparaison vaut à condition que l'on reconnaisse que le futur de la psychiatrie du jeune adulte reste largement ouvert. Il paraît en effet relativement clair à l'ensemble des chercheurs que les voies par lesquelles une réponse au problème de la schizophrénie sera obtenue sont très incertaines.

L'empirisme des programmes de recherche ou des propositions politiques suffirait à en témoigner. Du côté de la recherche la tendance actuelle est à la constitution par des consortiums internationaux de larges cohortes de patients auprès desquels pourront être recueillis un maximum de variables. Le recours à des méthodes d'analyse factorielle permet dans un second temps de tester un large spectre d'hypothèses sans avoir à en privilégier aucune d'emblée. De même, le constat de l'absence de recommandations fondées sur des preuves n'empêche pas les promoteurs de ces services de recommander d'expérimenter et de mettre en œuvre les initiatives les plus diverses pour éprouver ce qu'il en sortira. Le constat actuel est que, au fond, toutes les initiatives se valent.

\section{Des promesses multiples}

Les promesses des états mentaux à risques sont ainsi, encore une fois, plus indéterminée que ne veulent bien le dire leurs promoteurs. Des critiques issues notamment des rangs des sciences sociales ont pu en tirer la conclusion que ceux-ci étaient surdéterminés par une série de facteurs extrinsèques à la clinique : la montée du risque comme façon d'aborder un vaste ensemble de 
problèmes sociaux, l'émergence du jeune adulte comme nouvel âge de la vie, ou encore le passage de la psychiatrie à la santé mentale, notamment par le biais de l'extension des indications des traitements chimiothérapiques. (Rose, 2007; Moncrieff, 2013) C'est en somme pour de mauvaises raisons et en raison d'intérêts peu avouables qu'une partie significative de la psychiatrie se serait engagée dans la promotion de cette catégorie.

On peut cependant être tenté par une lecture moins stratégique. Le succès des états mentaux à risque tient en effet la façon dont ils ont acquis un sens pour un vaste ensemble d'acteurs aux origines les plus diverses. A cet égard la relative indétermination du concept pourrait être analysée comme une force plutôt qu'une faiblesse en ce qu'elle permet à chacun de le mobiliser au service de son propre agenda. On retrouve là des perspectives développées en sociologie des sciences autour du concept d'objet frontière pour caractériser des entités aux contours suffisamment souples pour pouvoir être mobilisés dans des mondes sociaux différents tout en état suffisamment robustes pour être utiles dans chacun de ces mondes (Star et Griesemer, 1989) : ainsi par exemple des outils classificatoires comme le DSM ou la CIM en psychiatrie dont l'insatisfaction qu'ils génèrent parmi les cliniciens peut s'expliquer précisément par l'utilité qu'ils présentent pour une multitude d'acteurs issus de mondes divers, de l'administration de la santé à l'assurance en passant par la recherche et la politique. Ces perspectives paraissent particulièrement fécondes si on ajoute à la caractérisation des objets frontières par leurs usages une dimension idéelle. C'est parce qu'ils rouvrent le champ des possibles dans une situation marquée a priori par le caractère implacable des échéances que les états mentaux à risque suscitent l'engagement.

Que ce soit dans la clinique ou au niveau disciplinaire les états mentaux à risque sont ainsi porteurs de perspectives d'autant plus riches qu'elles restent largement ouvertes. Jusqu'où se maintiendra l'engagement de la communauté psychiatrique derrière celles-ci c'est ce que l'avenir seul démontrera.

\section{Références}

Armstrong, D., et al., 2007, Health-related quality of life and the transformation of symptoms, Sociology of Health \& IIIness, 29, 4, 570-583.

Borck, C., 2009, Through the Looking Glass: Past Futures of Brain Research, Medicine Studies, 1, 4, 329-338.

Das-Munshi, J., et al., 2008, Public health significance of mixed anxiety and depression: beyond current classification, Br J Psychiatry, 192, 3, 171-177.

Fusar-Poli, P., et al., 2012, Predicting psychosis: meta-analysis of transition outcomes in individuals at high clinical risk, Arch Gen Psychiatry, 69, 3, 220-229.

Krebs, M. O. et Canceil, O., 2004, Détection précoce des troubles psychotiques: enjeux de la mise en place d'un réseau de soins, Revue Française des Affaires Sociales, 1, 91-102.

Lantéri-Laura, G., 1993, La sémiologie psychiatrique: son évolution et son état en 1982, In: Recherches psychiatriques. III Sur la sémiologie. Chilly-Mazarin, Sciences en Situation, pp. 465-497.

Marshall, M. et Rathbone, J., 2011, Early intervention for psychosis, Schizophr Bull, 37, 6, 1111-1114.

McGorry, P., 2010, Evidence, early intervention and the tipping point, Early Intervention in Psychiatry, 4, 1-3.

McGorry, P., 2012, At Issue: Cochrane, Early Intervention, and Mental Health Reform: Analysis, Paralysis, or Evidence-Informed Progress?, Schizophrenia Bulletin, 38, 2, 221-224.

McGorry, P. D., 2007, Issues for DSM-V: clinical staging: a heuristic pathway to valid nosology and safer, more effective treatment in psychiatry, Am J Psychiatry, 164, 6, 859-860.

Moncrieff, J., 2013, The bitterest pills : the troubling story of antipsychotic drugs, Palgrave Macmillan 
Petitjean, F. et Marie-Cardine, M., 2003, Schizophrénies débutantes : diagnostic et modalités thérapeutiques : conférence de consensus, 23-24 janvier 2003, Paris, Hôpital Sainte-Anne, Fédération française de psychiatrie, Montrouge, J. Libbey Eurotext.

Rose, N. S., 2007, The politics of Life Itself : Biomedicine, Power, and Subjectivity in the Twenty-First Century, Princeton, NJ, Princeton University Press

Ruhrmann, S., et al., 2010, Probably at-risk, but certainly ill--advocating the introduction of a psychosis spectrum disorder in DSM-V, Schizophrenia Research, 120, 1-3, 23-37.

Star, S. L. et Griesemer, J. R., 1989, Institutional Ecology, "Translations" and Boudary Objects. Amateurs and Professionals in Berkeley's Museum of Vertebrate Zoology, 1907-1939, Social studies of science, 19, 387-420.

Van Os, J. et Delespaul, P., 2005, Toward a world consensus on prevention of schizophrenia, Dialogues Clin Neurosci, 7, 1, 53-67.

Wakefield, J. C., 2009, Disability and diagnosis: should role impairment be eliminated from DSM/ICD diagnostic criteria?, World Psychiatry, 8, 2, 87-88.

Yung, A. R., et al., 1996, Monitoring and care of young people at incipient risk of psychosis, Schizophr Bull, 22, 2, 283-303.

Yung, A. R., et al., 2003, Psychosis prediction: 12-month follow up of a high-risk ("prodromal") group, Schizophrenia Research, 60, 1, 21-32.

Yung, A. R., et al., 2007, Declining transition rate in ultra high risk (prodromal) services: dilution or reduction of risk?, Schizophr Bull, 33, 3, 673-681.

Yung, A. R., et al., 2012, Whither the attenuated psychosis syndrome?, Schizophr Bull, 38, 6, 11301134. 


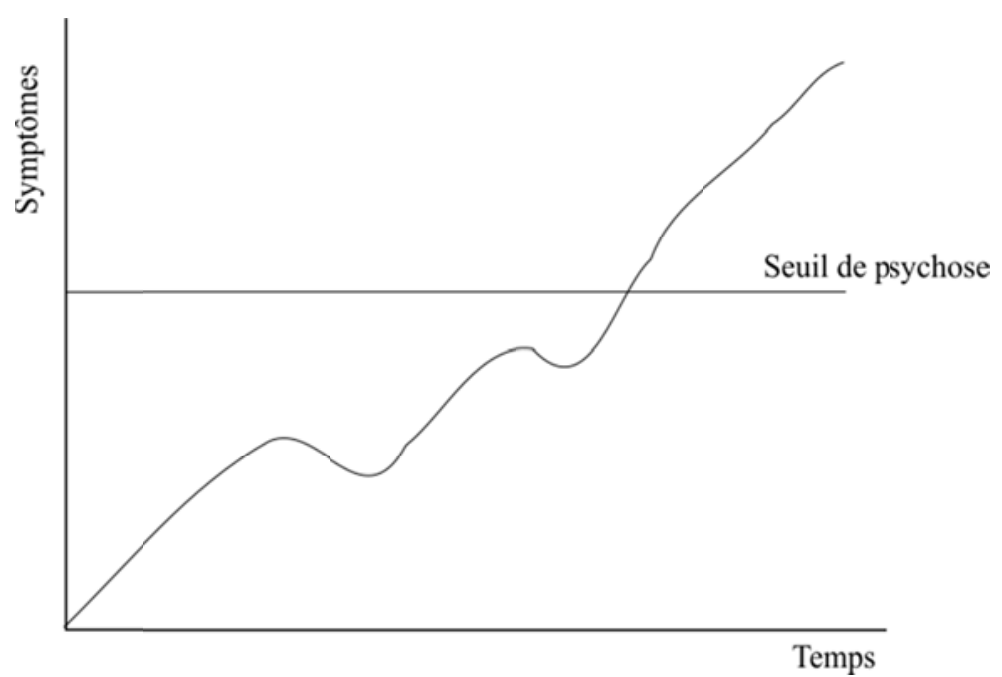

a. Vrai positif : le seuil de psychose est dépassé

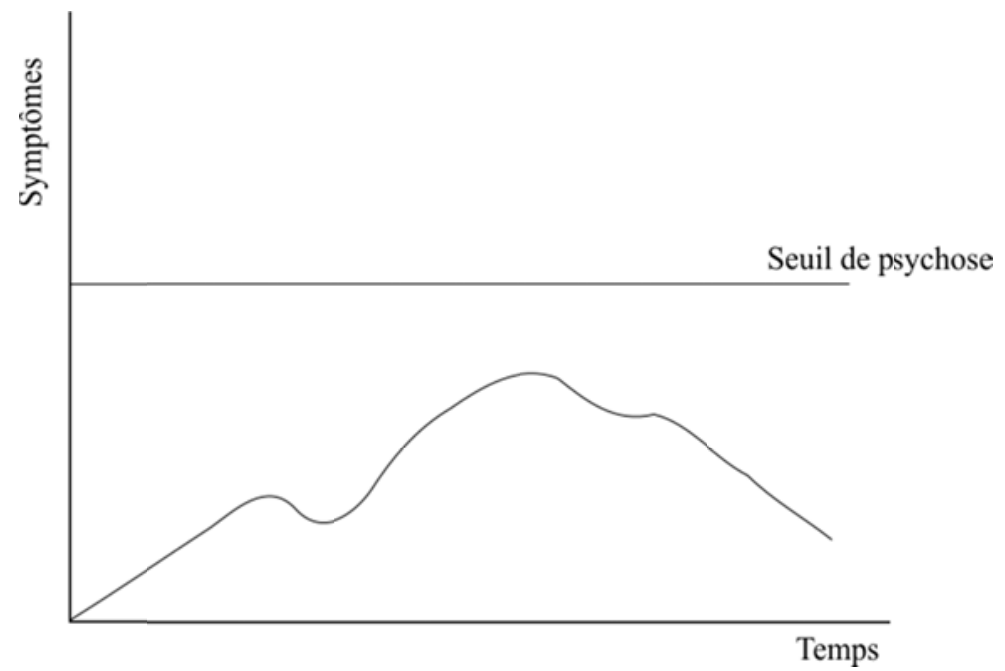

b. Faux positif : le seuil de psychose n'est pas dépassé

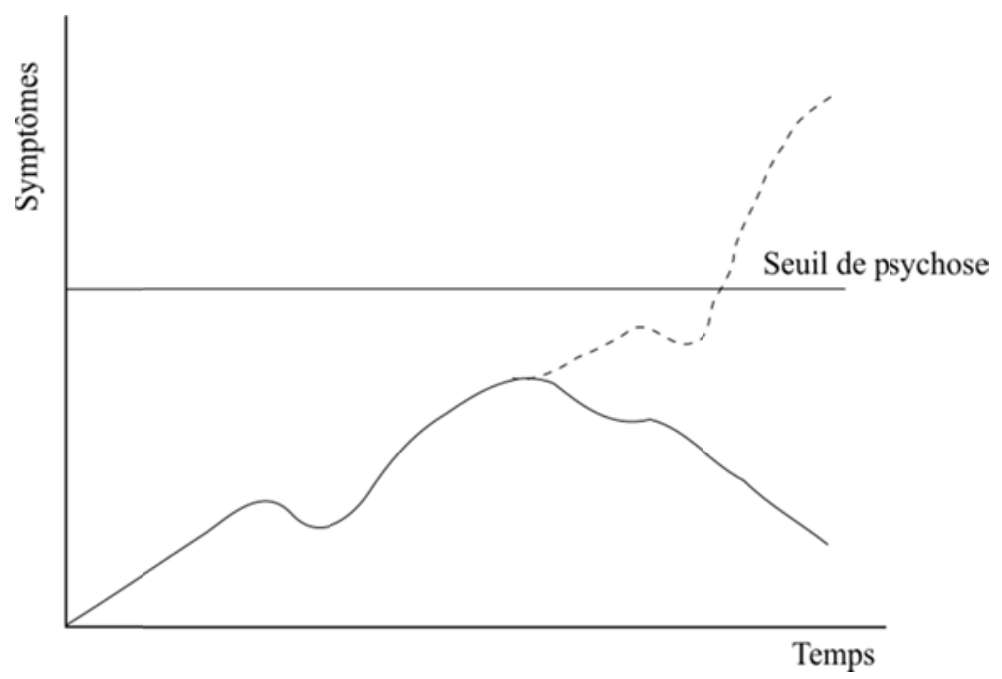

c. Faux faux positif : le seuil de psychose n'est pas dépassé, mais il l'aurait été sans intervention ou sous d'autres circonstances 
Fig. 1. Vrais, faux et faux faux positifs : les trajectoires imaginées de l'entrée dans la psychose. D’après Yung, A. R., H. P. Yuen, G. Berger, S. Francey, T. C. Hung, B. Nelson, L. Phillips, and P. McGorry. "Declining transition rate in ultra high risk (prodromal) services: dilution or reduction of risk?". Schizophr Bull 33, no. 3 (May 2007): 673-81. 